\title{
Effects of Two and Three-Dimensional Visual Objects on the Acquisition of Drawing Skills among JSS1 Students in Osun State, Nigeria
}

\author{
Bada Tayo Abass ${ }^{1, *}$, Bello Isyakka ${ }^{1}$, Ijisakin Yemi Olaolu ${ }^{2} \&$ Fajuyigbe Michael Olusegun ${ }^{2}$ \\ ${ }^{1}$ Department of Educational Technology, Obafemi Awolowo University, Ile- Ife, Nigeria \\ ${ }^{2}$ Department of Fine and Applied Arts, Obafemi Awolowo University, Ile-Ife, Nigeria \\ *Corresponding author: Department of Educational Technology, Obafemi Awolowo University, Ile- Ife, Nigeria. \\ E-mail: badatayo2@yahoo.com
}

Received: November 11, 2013

Accepted: December 20, 2013

Online Published: February 11, 2014

doi:10.5430/wje.v4n1p62

URL: http://dx.doi.org/10.5430/wje.v4n1p62

\begin{abstract}
The study examined the effects of two and three dimensional visual objects on learners' drawing skills in junior secondary schools in OsunState.Nigeria.It also determined students' ability to identify visual objects. Furthermore, it investigated the comparative effectiveness of two and three dimensional visual objects on drawing skills of junior secondary school students. The study employed the pretest, post- test experimental design. The study population consisted of all junior secondary school one students in Osun State. Three junior secondary schools were purposively selected from Atakumosa Local Government Area of Osun State. A sample of 120 JSS1 students was selected from the three schools using stratified sampling techniques with sex as stratum. These were later randomly assigned into two experimental and one control groups. The first experimental group was taught drawing using three-dimensional visual objects; the second experimental group was taught using two-dimensional visual objects, while group three the control group was taught using conventional method. The research instrument was a Visual Object Achievement Test (VOAT). This was administered as pre test and post test. Data gathered were analyzed using ANOVA and t-test statistics.The results showed that there was a significant difference in the basic drawing skills of learners taught with visual objects and those taught without visual objects $(\mathrm{F}=14.163, \mathrm{p}<0.05)$. The results also showed that there was a significant difference in the identification ability of learners taught with two and three dimensional visual objects and those taught with conventional method. $(\mathrm{F}=4.599, \mathrm{p}<0.05)$. The study concluded that the use of two and three dimensional objects will anhance drawing skills.

Furthermore, the results showed that there was no significant difference in the acquisition of drawing skills between male and female students who were taught using two and three dimensional visual objects $(t=2.02, p<0.05)$.

The study concluded that the use of two and three-dimensional visual objects significantly enhanced students' drawing ability.
\end{abstract}

Keywords: two dimensional objects, three dimensional objects, visual object

\section{Introduction}

Traditionally the term art was used to refer to any skill. This conception changed during the Romantic period when art came to be seen as "special faculty of the human mind which has to do with religion and science" (Gombrich, 2005).Ogumor (1990) stated that the most important function of art is that it is the visual representation of human thoughts or feeling, in forms of objects, (natural or man-made) human-animal being and the landscape in general, that satisfy one's craving for the good. He added that art is the expression of the characteristic of the people of an age or that it symbolizes an age, sect or group of people. The cross, for example, is a work of art and once you see somebody wearing it you will know that the person belongs to a certain group, that is.the Christians. The branches or areas of art include Painting, Graphics, Ceramics, Sculpture, Textiles, Architecture and Art History.However,the reality is that each aspect or area of art requires drawing.Drawing on other side could be taught to learners using two or three dimensional visual symbols. The word "Symbol" is operationally defined in this study as object with length, height and width which is also referred to as 3 Dimensional, and object with length and width which is referred to as 2 
Dimensional (Suzanne, 2006). The importance of teaching Fine Arts in Nigeria secondary schools cannot be over emphasized. Fine Arts is a subject that deals with culture and artistic creation of the past, present and future. Fine Arts as a subject explains the significance of art works to humanity, taking into consideration the style of execution, time and period of production. Gustafson (1993) stated that, "the future belongs to those who respect the past, acknowledge the present, and grasp the future". It is a common saying that art speaks, but who can understand its language? Art is more than a form of communication. Artists in every age express themselves intelligently and creatively through drawing. The meaning of their works can clearly be understood when two or more of such works are clearly drawn and produced at almost the same time and compared. According to Dibujos (2009), drawing is a means of making an image, using any of wide variety of tools and techniques. It generally involves making marks on a surface by applying pressure from a tool, or moving a tool across a surface using dry media such as graphite pencils, pen, and ink, inked brushes, wax colour pencils, crayons, charcoals, pastels, and markers. The main techniques used in drawing are: line drawing, hatching, and crosshatching, random hatching, scribbling, and blending. It may become increasingly difficult for learners to apply these techniques in drawing if attention is not adequately directed to the educational system that will encourage the teaching and learning of Fine Arts in Junior Secondary Schools. However, Bada (2003) stated that the number of students that enrolled for the Fine Arts in schools keep decreasing every year and most probably with no student enrolment to sit for Senior Secondary School Fine Arts Examinations in some schools. Over some years, it is observed that the development of art teaching in some schools, both at the State and Federal level is deplorable. There are shortages of art teachers in secondary schools. In most cases the few teachers available are unprofessional and therefore could not teach adequately with relevant materials. Fadare, (1986) also observed that Fine Arts as a subjects lacks materials such as 2 dimensional and 3 dimensional visual objects, charts, display boards, films, and filmstrips, slides, video, and other materials that could facilitate learning. In schools where these materials are available they are hardly used. Some have nothing to show or exhibit, some could not even boast of an art gallery or studio and many students were not exposed to museums while participation in art exhibition is rarely encouraged. According to Anyasode (2010) in Bada, (2013) some of the problems in Fine arts include the following:

- non recognition of Fine Arts by the parents and the government;

- non acceptability of the subject in the Nigerian situation by the public;

- poor attitude of principals in secondary schools towards the subject and Fine Arts students;

- failure of policy-making organs of the government to give Fine Arts prominence in the educational system.

The researchers observed that art in both primary and secondary schools are not given adequate attention and recognition it deserved owning to the negative attitude of the school administrators towards the subject. It is also observed in some secondary schools within Osun State, Nigeria that most students have negative attitude towards art subject. This may be due to parental negative influence against Fine Arts. More attention are given to subjects such as English, Mathematics, Social Studies, Integrated Science, and all other subjects above Fine Arts.

Despite all the problems facing Fine Arts education in our schools, it has been observed that society and the school community seldom do without the input of a professional artist to solve some problems of aesthetic degradation in our environment. According to David-West (1992) the global culture is astigmatic, hence they do not indicate the idea that creative arts are indeed fundamental to human growth. In view of the highlighted problems by art educators, the need to examine what is presently going on in our society and of course in our schools becomes inevitable if the following objectives as stated in the West African Examinations Council $(1990,1991)$ are to be achieved. The objectives are stated thus:

(a) Development of perceptual, analytical and expressive skills through a variety of art activities relating to the environment.

(b) Acquisition of knowledge and skills in the use and maintenance of art tools, equipment and materials.

(c) Attain the level of appreciation of values and qualities of different works of art.

(d) Acquisition of knowledge and application of design elements and principles.

(e) Acquisition of the knowledge of Art History and Art structure.

(f) Acquisition of the knowledge and appreciation of their culture in relation to other cultures',

(g) Understanding of the meaning, significance and role of art in the socio-economic development of their society.

(h) Understanding and development of indigenous art technology, aesthetic, beliefs, values and attitudes. 
In view of the need to implement these objectives, the methods of instruction to be employed by art teachers need re-examination. It is observed that Art teachers use expository and exploratory methods. Here there are three ways interactions: the teacher interactions, with the students, the students interact with the materials and the student-teacher also interacts with the materials. These two methods are very extensive that the significance of 2 dimensional and 3 dimensional visual objects cannot be underestimated. Society is moving into a new era of rapid change and by ongoing advancements in the use of teaching materials for orientation, information, teaching, and better communication. The present and of course the future learners require different kinds of skills and materials that will teach them better. Our school environment therefore should be an opportunity to explore and determine their goals through generous access to instructional materials. In this regard, the use of visual objects becomes necessary for students' in order to understand and assimilate various concepts being taught in our secondary schools. Hoban (1951) in his own opinion stated that the use of visual materials would enhance the performance of students in the classroom. As a result of the inherent attributes of visual materials, they are very relevant for instructional use. Jean (1993) was of the opinion that visual materials can be used to teach, and through the visual materials, social events can be dramatized and historical personage can be brought to the classroom. When visual objects are used in the teaching-learning situation, the feelings and emotion of the student are arrested. Visual materials affect and induce the students' interest and sense of participation. Paul (1990) stated that research studies have shown that visual object has great impact on emotion. According to him, this attribute makes it relevant to the affective domain of learning. He stresses that visual objects can be used to command and arrest students attention. In the same vein, Henry and Phil (1993) stated that visual objects are sort of supportive and illustrative materials for use in expository teaching.

On the issue of performance that whether male or female will be significantly better when exposed to visual objects, educational research has since been controversial and non-conclusive. It has always been argued that illiteracy rates are higher among females than males in every part of the world (Bank, 1988). Research findings sometimes indicated significant sex differences while some others could not support the idea of sex factor influence on learners performance. Bank, (1988) in an extensive preview of research held that boys generally achieved higher scores than girls in mathematics and the physical sciences. Boys in most countries outperform girls in biology but girls in Wales, England and New Zeland achieve higher scores in this subject. Girls in most part of the world achieve higher scores than boys in Literature and French as a foreign language.

Thompson (1975) concluded that in reading attainments among children, there is no difference between reading of boys and girls ten years of age and older, but under ten years of age mean attainment have been found lower for boys than girls. However, Fairweather, (1976) opined that most studies indicating sex differences may be the result of sex-role stereotypes influence. In the same vain, Sek and Usis (1983) noted that findings from recent studies indicated that boys and girls perform equally well in complex tasks even in mathematics if both out-of-the class and in-the-class exposure to any given task is equal. They asserted further that when experience can be controlled regardless of the difficulty or complexity of the item girls and boys performed equally well.

The study of Owen and Colvein (1979) revealed that regardless of visual format used, males achieved significantly higher than females. Eccleston, Bokin, and Burrows (1990) also examined the performance of boys and girls at General Certificate in Education, Advance Level (GCE A/L) over a four period in a British sixth form college students General Certificate in Education Ordinary and 'A'Level results were quantified for the research. Product moment correlation co-efficient between 'O' Level score per candidate against the aggregate was calculated.

Index calculated reveals that boys out-performed the girls in all subjects except English Language and Literature. However, differences in a grade for individual subjects were not statistically significant. In English Literature, Economics, Mathematics, Physics, Chemistry, Geology, and Environmental sciences, boys achieved higher 'A' Level scores. In History, where 'O' Level scores of boys and girls are compatible, the boys achieved a whole grade point better than girls at 'A' Level on average. In another study, Yusuf (1991) examines the influence of sex on performance of students taught using flat pictures. Results of the null hypothesis tested for sex using t-test (tc $=86 ; \mathrm{df}=148, \mathrm{P}$. >.05) indicated no significant level. The result indicated that both male and female students benefited equally through the use of flat pictures in social studies instruction. Clerg and Simonson (1975) in a review of educational media research in relation to sex variable opined that most researches on media were based on samples that contained exclusively male subjects and yet generalizations of results were made to two sex population. Their review revealed that a disproportionate number of authors did not report an examination of sex difference and of the studies that evaluated on media use, 64\% found significant difference between male and female subjects on criterion measurement. Finally, most of the researchers reported that sex, as a variable, needed closer scrutiny. They concluded that reporting of sex difference information should become a standard procedure for researchers evaluating the effectiveness and applicability of media. 


\section{Objectives of the Study}

The objectives are to

(i) examine the effect of two and three dimensional visual objects on learners' skills in basic drawing.

(j) determine students ability to identify visual objects.

(k) compare the effectiveness of two and three dimensional visual objects on the acquisition of drawing skills of male and female students.

\section{Research Hypotheses}

The following hypotheses were generated for this study.

(1) There will be no significant difference in the drawing skills of learners taught with visual objects and those taught without.

(2) There will be no significant difference in the ability to identify objects by the learners taught with two dimensional and three dimensional visual objects and those taught without.

(3) There will be no significant difference in the acquisition ofdrawing skills of male and female students taught with two and three dimensional visual objects

\section{Research Methodology}

\subsection{Population}

The population of this study was made up of Junior Secondary School One (JSS 1) students in Atakomasa Local Government.

\subsection{Sample}

Three schools were purposively selected from thirteen schools. The selection was based on availability of Fine art studio and schools that have continually teach Fine Art for 10 years. One hundred and twenty JSS I students were randomly selected from three schools`.

\subsection{Research Design and Treatment}

This study adopted the pre-test, post-test control group (Campbel and Stanley, 1972). This design entails the random assignment into two experimental and one control groups. Both groups were exposed to a practical drawing exercise. Students in experimental group ' $A$ ' were exposed to Three Dimensional Visual Objects and experimental group 'B' were exposed to Two Dimensional Visual Objects. The Control group was taught using conventional classroom method.

\subsection{Instrument}

The instrument used was Visual Objects Achievement Test (VOAT). This was a fifteen item objective questions to evaluate the level of mastery of content taught. They were later asked to draw from two-dimensional and three-dimensional visual objects.

\subsection{Data Gathering Procedure}

The experimental group was taught using two dimensional and three dimensional visual objects. On the other hand the control group was exposed to the conventional method, that is, without using two dimensional and three dimensional visual objects. However, both experimental and control groups were exposed to the same contents and the same number of lessons. the students wherever they have difficulties. The first lesson was used for the pretest. The experimental and control group were exposed to the pre-test in order to determine their previous knowledge. During the second week, the teacher introduced two dimensional and three dimensional visual objects and what is expected of the groups. The same thing was applicable to males and females students. The control group was also taught but without using two dimensional and three dimensional visual objects.

\section{Results}

In testing the hypotheses for this study, two types of data were involved. These were data collected during the pre-test and posttest administrations of the research instrument. 


\subsection{Hypothesis One}

This hypothesis states that there will be no significant difference in the drawing skills of learnerstaught with visual objects and those taught without.

To test this hypothesis, the pre-test and posttest scores of subjects in the two experimental groups, that is, three-Dimensional Visual Objects (TDVO) and Two-Dimensional Visual Objects (TWDVO) and control group were subjected to one-way Analysis of Variance (ANOVA) and Analysis of co-variance. (ANCOVA) the results are presented in Tables 1 and 2

Table 1. Pretest and Post-test Means and Standard Deviation Scores of Subjects in Experimental and Control Groups

\begin{tabular}{lllll}
\hline TEST & GROUP & N & MEAN SCORES & SD \\
\hline PRE-TEST & TDVO (3-D) & 40 & 9.2 & 2.2284 \\
& TWDVO (2-D) & 40 & 8.9 & 1.0592 \\
& CONTROL & 40 & 8.6 & 1.0256 \\
POST-TEST & TOTAL & 120 & 8.9 & 1.4377 \\
& TDVO (3-D) & 40 & 10.375 & 1.576 \\
& TWDVO (2-D) & 40 & 9.250 & 0.893 \\
& CONTROL & 40 & 6.387 & 6.0177 \\
& TOTAL & 120 & 8.636 & \\
\hline
\end{tabular}

Table 2. ANCOVA

\begin{tabular}{llllll}
\hline \multicolumn{1}{c}{ SOURCE } & $\begin{array}{c}\text { SUM OF } \\
\text { SQUARES }\end{array}$ & df & MEAN SCORES & F & SIG. \\
\hline Corrected model & 62.024 & 2 & 31.012 & 7.179 & .001 \\
Intercept & 579.360 & 1 & 579.360 & 134.110 & .000 \\
Pretest & .008 & 1 & .008 & 0.02 & .967 \\
Visual/object group & 61.183 & 1 & 61.183 & 14.163 & .000 \\
Error & 505.442 & 117 & 4.320 & & \\
Total & 13216.000 & 120 & & & \\
Corrected total & 567.467 & 119 & & & \\
\hline
\end{tabular}

From Table 1, subjects in the TDVO group had a pre-test means score of 9.2 in the achievement test with a standard deviation of 2.2284 while subjects in the TWDVO group had a mean value of 8.9 and a standard deviation of 1.0392. Also, subjects in the control group had a mean score of 8.6 and a standard deviation of 1.0256 at pre-test level. When these values were subjected to Analysis of co-variance (ANCOVA) as shown in Table 1.1 an F-ratio of 0.02 was obtained. This value is not significant at 0.05 level. This implies that prior to the treatment programme, the subjects in the three groups were not statistically different in their drawing skills.

However, as shown in Table 1 the posttest means scores of subjects in the TDVO was 10.375 with a standard deviation of 1.576. also, in the TWDVO group, a post-test mean score of 6.3857 and a standard deviation of 3.0127 were obtained. Subjects in the control group however had a post-mean score of 9.25 and a standard deviation of 0.893 . As shown in Table 1.1, these values yielded an F-ratio of 14.163 which is significant at 0.05 level. This suggests that the post-test mean scores of subjects in the groups were statistically different.

Table 3. Multiple Comparison of Mean Values of the Performance of Subjects in Experimental and Control Groups

\begin{tabular}{llllll}
\hline Group & $\mathbf{N}$ & $\mathbf{X}$ & $\mathbf{S . D}$ & $\begin{array}{l}\text { Mean } \\
\text { difference }\end{array}$ & $\mathbf{P}$ \\
\hline TDVO (3-D) & 40 & 10.375 & 1.576 & & \\
TWDVO (2-D) & 40 & 9.25 & 0.893 & 1.125 & $<0.05$ \\
TDVO (3-D) & 40 & 10.375 & 1.576 & & $<0.05$ \\
Control & 40 & 6.385 & 3.0177 & 1.4417 & $<0.05$ \\
TWDVS(2-D) & & 9.25 & 0.893 & & 2.865 \\
CONTROL & 40 & 6.385 & 3.0177 & & $<$ \\
\hline
\end{tabular}


From the Table 3, the mean difference between the TDVO and TWDVO groups is 1.125 while the mean difference between the TDVO and control is 1.4417. Also, the mean difference between the TWDVO and control group is 2.865 . these differences are all significant at 0.05 level, which suggests that the differences in the post-test performance of subjects in the three groups were statistically significant. It will also be observed that the TDVO group had the highest post mean score followed by the TWDVO group while the control group had the least score. The summary of the foregoing analysis is that the performance of subjects in the three dimensional visual objects group is superior to that of the two dimensional visual objects group. However, subjects in the two experimental groups out-performed their colleagues in the control group. The implication therefore, is that an effective use of visual objects

whether three dimensional or two dimensional would enhance teaching and facilitate the acquisition of drawing skills.

\subsection{Hypothesis Two}

The hypothesis states that there will be no significant difference in the ability to identify visual objects by the learners taught with two dimensional and three dimensional visual objects and those taught without. The result of the analysis of the data collected in respect of this hypothesis is presented in table 4 and 5.

Table 4. Mean and standard Deviation of Subjects ability to identify Visual Objects in Experimental and Control Groups

\begin{tabular}{llll}
\hline Group & N & Mean & SD \\
\hline Two Dimensional & 40 & 10.7750 & 1.95445 \\
Three Dimensional & 40 & 11.3500 & 1.88856 \\
Control & 40 & 9.9500 & 2.35285 \\
Total & 120 & 10.6917 & 2.13729 \\
\hline
\end{tabular}

Table 5. Differences in the Ability Scores to Identify Visual Objects of Subjects in Experimental and Control Groups

\begin{tabular}{llllll}
\hline Source of variance & Sum of squares & df & Means square & F & Sig. \\
\hline Between groups & 39.617 & 2 & 19.808 & 4.599 & .012 \\
Within groups & 503.975 & 117 & 4.307 & & \\
Total & 543.592 & 119 & & & \\
\hline
\end{tabular}

From table 4 subjects in Two Dimensional group had a mean score of 10.7750 and a standard deviation of 1.95445 and Three Dimensional group had a mean score of 11.3500 and a standard deviation of 1.88856. Also subjects in the control group had a mean score of 9.9500 and standard deviation of 2.35285 subjected to one way Analysis of Variance as shown in table 5 an F-ratio of 4.599 was obtained. This value is significant at 0.05 level. This implies that there is a significant difference in the ability to identify visual objects in the experimental and control groups. Thus, the hypothesis which states that there will be no significant difference in the ability to identify visual objects by the learners taught with two dimensional and three dimensional visual objects and those taught without is rejected.

In order to find out which of the groups had higher scores in the ability to identify visual symbols after the treatment, the mean scores of subjects is the three groups were subjected to a post-hoc multiple comparison test using the least significant difference formula. The results are as shown in Table 6

Table 6. Multiple Comparison of Mean Values of Subjects' Ability to Identify Visual Objects in Experimental and Control Groups

\begin{tabular}{lllll}
\hline $\begin{array}{l}\text { Experimental } \\
\text { group (i) }\end{array}$ & $\begin{array}{l}\text { Experimental } \\
\text { Group (j) }\end{array}$ & Mean difference (i-j) & Std error & Sign. \\
\hline Two D & Three-Dimension control & -57500 & 1.46408 & .466 \\
& & .82500 & .46408 & .210 \\
Three D & Two-dimension & .57500 & .46408 & .466 \\
& control & 1.40000 & .46408 & .013 \\
Control & Two - D & -.82500 & .46408 & .210 \\
& Three -D & -1.40000 & .48408 & .013 \\
\hline
\end{tabular}

(The mean difference is significant at the 0.05 level)

From table 10.2.3, the ability to identify visual object mean difference between the two dimensional visual object group and three-dimensional visual object group is -.57500 while the ability to identify visual object between three dimensional visual object group and control group is .82500 .. The ability to identify visual object mean difference between control group and two dimensional visual object group is -.82500 while the ability to identify visual objects 
between control group and three dimensional visual object group is 1.40000 . These mean differences are significant at 0.05 level, which suggests that the differences in the abilities to identify visual objects of learners in the three groups were statistically significant. It is also observed that students exposed to three dimensional visual objects had the highest mean scores followed by those exposed to two dimensional visual objects while those taught using the conventional classroom teaching method (control group) had the least ability to identify visual objects mean score. From the foregoing, it can be concluded that the use of visual objects, either three dimensional or two dimensional visual objects would enhance learners' ability to identify visual objects more than the use of the conventional classroom teaching method.

\subsection{Hypothesis Three}

The hypothesis states that there will be no significant difference in the acquisition of drawing skills among male and female students who are taught using two dimensional and three dimensional visual objects. To test this hypothesis, the performance of male and female students was subjected to descriptive analysis. The results are presented in Table 7.

Table 7. Differences in the Performance of Subjects According to Gender

\begin{tabular}{lllll}
\hline Students sex & Experimental treatment & Mean & Std. Deviation & N \\
\hline Male & Three-dimensional & 11.2500 & 2.67296 & 20 \\
& Two-dimensional & 10.7000 & 2.69698 & 20 \\
\multirow{4}{*}{ Female } & Total & 10.9750 & 2.66494 & 40 \\
& Three dimensional & 10.6000 & 1.95744 & 20 \\
\multirow{3}{*}{ Total } & Two-dimensional & 9.6500 & 1.66307 & 20 \\
& Total & 10.1250 & 1.85621 & 40 \\
& Three-dimensional & 10.9250 & 2.33576 & 40 \\
& Two-dimensional & 10.1750 & 2.27458 & 40 \\
& Total & 10.550 & 2.32161 & 80 \\
\hline
\end{tabular}

From Table 7. The mean score of male subjects in Three-Dimensional Visual objects is 11.2500 and standard deviation of 2.67296 while the mean score of male subjects in Two Dimensional Visual objects is 10.7000 and standard deviation of 2.69698 which reveals that there is no significant difference in the use of two dimensional and three-dimensional visual objects among male subjects after treatment. The mean score of female subjects in three dimensional visual object is 10.6000 and standard deviation of 1.95744, while the mean score of female subjects in two-dimensional visual object is 9.6500 and standard deviation of 1.66307. The mean score and standard deviation obtained here also show that there is no significant difference in the acquisition of drawing skills using two dimensional and three-dimensional visual objects among female students. After treatment, the mean scores and standard deviation which reveals no significant difference shows that gender is not a factor. The mean score of both male and female subjects in three dimensional visual objects is 10.92500 and standard deviation of 2.38596. Also, the mean score of male and female students in two-dimensional visual objects is 10.1750 and standard deviation of 2.27458. This shows that there is no significant difference in the acquisition of drawing skills between male and female students who were taught using two dimensional and three-dimensional visual objects.

\section{Discussion}

This section presents a discussion of the major findings of the study in respect of each of the research hypotheses.

\subsection{Effect of two Dimensional and Three Dimensional Visual Objects on Learners'Drawing Skills}

Hypothesis one was tested to examine the effect of two and three dimensional visual objects on learners' drawing skills. The results revealed that there was a significant difference in the post-test mean scores of the students exposed to the three methods. It was also revealed that the three dimensional visual objects group had the highest post-mean scores followed by the two-dimensional visual objects group while the control group had the least score. The superiority of the three-dimensional group over two dimensional and conventional method could be as a result of the importance of three-dimensional visual objects in the acquisition of drawing skills. However, it is not surprising that this study has shown that the visual objects either three dimensional or two dimensional visual objects has a significant positive effect on students' performance in drawing. This is because the visual objects ensures that the instructional content is more real and delivered with vigour. Vigour implies conciseness with precision, as subtle idea can be expressed with faintest shades of meanings revealed through visual objects. This findings are in consonance with the results of previous 
studies by Donald (1983), Yusuf (1991), Gareth (1988), Nelson (1980) Sofowora (2001) and Adeyanju (2001). These researchers had found out that stimulus materials could enhance significantly the performance of learners.

6.2 Influence of Two Dimensional and Three Dimensional Visual Objects on Learners Ability to Identify Visual Objects

Hypothesis 2 was used to test for significant difference in the ability to identify visual objects mean scores of students taught using three-dimensional, and two-dimensional visual objects and those taught without. Findings from this hypothesis showed that students that were exposed to both three dimensional and two-dimensional visual objects performed significantly better because some factors that caused loss of the ability to identify visual objects had been reduced by the use of both three dimensional and two dimensional visual objects. Some of these factors include visual presentation, motivation and interest. The students that were exposed to the visual objects had a chance of not only to identify visual objects but to also represent them in drawing this resulted in reinforcement of instruction and hence the increase in their performance and ability to identify visual objects.

Adeyanju (1997) in a study on the effects of repetition of audio-taped instruction and visuals on acquisition of cognitive imagery found that there was an increase in the drawing skills of students when a combination of Yoruba and English was used along with the enrichment segment. Similarly, Valentine (1960) claimed that anything which arouses attention and same level of concentration leaves some expression and that the greater the interest aroused, the stronger the ability to identify visual objects. The implication of this finding is that three dimensional and two dimensional visual objects if effectively used in instruction particularly in drawing can help improve significantly the ability to identify visual objects and develop the acquisition of drawing skills.

6.3 Effect of two with Three Dimensional Visual Objects in the Acquisition of Drawing Skills among Male and Female Students

Hypothesis 3 was used to test for the significant difference in the acquisition of drawing skills among male and female students who are taught using two dimensional with three dimensional visual objects. The result of the analysis of hypothesis 3 shows in table 10.3.1 revealed that there was no significant difference when two dimensional and three dimensional visual objects were used to teach basic drawing skills among the male subjects. The three dimensional and two dimensional visual objectls were also administered to the female students. The result shows that there is no significant difference in the mean score when the three dimensional and two dimensional visual objects were presented.

After treatment, the mean score and standard deviation which reveals no significant difference shows that gender is not a factor. When both male and female were tested, their mean score reveals that there is no significant difference in the acquisition of drawing skills among male and female students who were taught using two dimensional and three-dimensional visual objects.

\section{Conclusion}

This study concludes that the use of two and three dimensional objects would produce a significant improvement in drawing skills ability of students, in addition the use of either two or three dimensional objects in drawing exercise will increase students' ability to identify ability visual objects.

\section{References}

Adeyanju, J.L. (2000). Basic concept in Educational Technology: A resource handbook for In-service and Pre-service teachers in West African Countries; University of Education, Ghana.

Anyasode, B.A. (2010). Religion and the society. Journal of Society, 11, 75-90.

Bada, T.A. (2013). Towards Effective Utilization of the Mass Media to Improve the Quality of Instruction in Schools. Nigeria Journal of Social Sciences, 3, 148-159

Bank, D.O. (1988). Health Education in Developing Countries. London: Nelson Bookshed.

David-West, T. (1992). Toward Integration: Education, Instructional Technology, and Semiotics. Educational Communication and Technology Journal, 20(2), 75-89.

Dibujos, B. A. (2009). Religion and the Growing Mind. Journal of Educational Review, 11, 75-90.

Donald, S. (1983). Interactive Update. Interactive Video in Language Teaching, 15(7), 50-55. 
Eccleston, G., Bokin, I., \& Burrows, A. (1990). Sex Related Differences in Academic Performance at GCE Level. Educational Research, 32(3), 229-232. http://dx.doi.org/10.1080/0013188900320311

Fadare, D.O. (1986). Arts and Society. Ibadan: Onibonoje Press.

Fairweather, H. (1976). Sex Differences in Cognition. Cognition, 4, 231-280.

Gareth, A. C. (1988). Help to Draw in Developing Countries. London: Nelson Press.

Gombrich, T.P. (2005). Techniques of Drawing in Nigerian Secondary Schools. Lagos: Macmillan.

Gustafson, F.D. (1993). Arts and Visual Objects. Ife: Omowumi Press.

Henry, T., \& Phil, L. (1993). Visual Education. Chicago: Graw-Hill Book company.

Hoban, C.F. (1951). Visualizing the curriculum. New York: Doyden.

Jean, M. (1993). Rethinking Interactivity, Lessons from Interactive Radio Instruction. Development Communication Report, 81(2), 9-11.

Nelson, Z. (1980). Interactive Programmed Design for Corporate Training. Education and Industrial Television, 5(2), 53-47.

Ogumor, M. (1990). Arts and Crafts in Nigeria Secondary Schools. Benin: Ozas Press.

Owen, R.D., \& Coldevin, G.O. (1979). Effects of Varied Temporal Overlapping in Multi-image Tape - Slider Presentation. Programmed Learning and Educational Technology, 14(1), 33-42.

Paul, B. (1990). Educational and Training Technology International. Interactive Video and Language Learning, 27(2), 146-173.

Senks, S., \& Usiz, Z. (1983). Germany Proof Writing: A new view of sex differences in Mathematics ability. American Journal of Education, 91(2), 187-201. http://dx.doi.org/10.1086/443680

Suzanne, E. (2009). Learning Arts with Visual Objects. Onitsa: Ogudu Press.

Thompson, G. B. (1975). Sex Differences in reading attainment. Educational Research, 18(1), 16-23. http://dx.doi.org/10.1080/0013188750180102

West African Examination Council. (1990). Annual Report. Nigeria: National Press.

Yusuf, M. (1991). Learning with Flat Pictures. Osogbo: Oxford Press. 